\title{
Introducing model uncertainty by moving blocks bootstrap
}

\author{
Andrés M. Alonso ${ }^{1,2}$, Daniel Peña ${ }^{2}$, Juan Romo ${ }^{2}$ \\ 1 Departamento de Matemáticas, Universidad Autonóma de Madrid, 28049 Madrid, \\ Spain. e-mail: andres.alonso@uam.es \\ 2 Departamento de Estadística, Universidad Carlos III de Madrid, 28903 Getafe, \\ Spain. e-mail: \{amalonso, dpena, romo\}@est-econ.uc3m.es
}

It is common in parametric bootstrap to select the model from the data, and then treat as if it were the true model. Chatfield $(1993,1996)$ has shown that ignoring the model uncertainty may seriously undermine the coverage accuracy of prediction intervals. In this paper, we propose a method based on moving block bootstrap for introducing the model selection step in the resampling algorithm. We present a Monte Carlo study comparing the finite sample properties of the proposed method with those of alternative methods in the case of prediction intervals.

AMS (2000): 62M10, 62F 40.

Key words: sieve bootstrap, blockwise bootstrap, prediction, time series, model uncertainty.

\section{Introduction}

When studying a time series, one of the main goals is the estimation of forecast intervals based on an observed sample path of the process. The 
traditional approach to finding prediction intervals assumes that the series $\left\{X_{t}\right\}_{t \in \mathbb{Z}}$ follows a linear finite dimensional model with a known errors distribution, e.g. a Gaussian autoregressive-moving average $\operatorname{ARMA}(p, q)$ model as in Box et al. (1994). In such a case, if the orders $p$ and $q$ are known, a maximum likelihood procedure could be employed for estimating the parameters and then, plug in those estimates in the linear predictors. In addition, some bootstrap approaches have been proposed in order to avoid the use of a specified errors distribution, see e.g. Stine (1987) and Thombs and Schucany (1990) for $\operatorname{AR}(p)$ models, Pascual et al. (2001) for $\operatorname{ARMA}(p, q)$ models, and Alonso et al. (2002) for linear processes that admit a one-sided infinite-order moving average representation

$$
X_{t}-\mu_{X}=\sum_{j=0}^{+\infty} \psi_{j} \varepsilon_{t-j}, \quad \psi_{0}=1, t \in \mathbb{Z}
$$

where $\left\{\varepsilon_{t}\right\}_{t \in \mathbb{Z}}$ is a sequence of uncorrelated random variables with $\mathrm{E}\left[\varepsilon_{t}\right]=0$, $\mathrm{E}\left[\varepsilon_{t}^{2}\right]=\sigma^{2}$ and with at most a polynomial decay of the coefficients $\left\{\psi_{j}\right\}_{j=0}^{+\infty}$.

But, those bootstrap proposals assume that $p$ and $q$ are known or, in the case of Alonso et al. (2002), it selects an approximating autoregressive model $\operatorname{AR}(\widehat{p})$ from the data, and then use the selected order, $\widehat{p}$, as if it were the true order. Then, those approaches ignore the variability involved in model selection, which can be a considerable part of the overall uncertainty (e.g. Chatfield 1993, 1996). In practice, having observed a sample of size $n$, the model, and particularly $p$ and $q$ are invariably unknown. Thus, we should select a model from the data. Many model selection procedures have been proposed, e.g. the final prediction error (FPE) of Akaike (1969), the Akaike (1973) information criterion (AIC) or its bias-corrected version (AICC) of Hurvich and Tsai (1989) and the Bayesian information criterion of Schwarz (1978), see Bhansali (1993) for a review.

For finite autoregressive models, Masarotto (1990) and Grigoletto (1998), propose to take into account model uncertainty as follows: first, to obtain 
$\widehat{p}$ by a consistent model selection procedure, then generate bootstrap resamples from the estimated $\operatorname{AR}(\widehat{p})$ and to re-estimate in each resample the order by the same method used for $\widehat{p}$. Essentially the same algorithm was suggested by Kilian (1998) in the context of generating impulse response confidence intervals, the so called endogenous lag order bootstrap. Alonso et al. (2004) proposes a sieve endogenous order bootstrap for processes that admit the moving average representation (1). Also, Alonso et al. (2004) proposes a sieve exogenous order bootstrap based on the probability function for $\widehat{p}$ calculated using the values of the objective function of the information criterions (AIC, AICC, or BIC).

In this paper we propose a new method for introducing the sampling variability of the model selection procedure that is less dependent on $\widehat{p}$. This approach use an estimator of the distribution of $\widehat{p}$ based on moving block bootstrap. As in the sieve exogenous order bootstrap, once we have an estimated distribution $\widehat{F}_{p}$, we generate resamples from the estimated $\operatorname{AR}\left(p^{*}\right)$ with the $p^{*}$ i.i.d. $\widehat{F}_{p}$, and then we proceed as in standard bootstrap approaches.

The remaining of this paper is organized as follows. Section 2 presents the sieve exogenous order bootstrap based on blockwise bootstrap. In this section, we briefly present the moving missing block bootstrap and jackknife. In Section 3, we present the results of a Monte Carlo study comparing the finite sample properties of the proposed method with those of alternative methods.

\section{The sieve exogenous order bootstrap based on blockwise bootstrap}

2.1 Moving block resampling order distribution.

The moving block jackknife and bootstrap were introduced by Künsch (1989) and independently by Liu and Singh (1992). In the following, we use 
the presentation of Liu and Singh (1992): Lets $X_{1}, X_{2}, \ldots, X_{n}$ be random variables with the common distribution function $F_{X}$, and let $T$ be the parameter of interest and $T_{n}$ its estimator based on $\mathbf{X}=\left(X_{1}, X_{2}, \ldots, X_{n}\right)$. Let $B_{i}$ denote a block of $\ell$ consecutive observations, i.e. $B_{i}=\left(X_{i}, X_{i+1}, \ldots\right.$, $\left.X_{i+\ell+1}\right)$ for $i=1,2, \ldots, n-\ell+1$. Then,

- For the moving block jackknife (MBJ), we denote the $i$-th jackknife statistics by $T_{n,-i}$ which is equal to the estimator $T_{n-\ell}$ evaluated in the reduced sample $\mathbf{X} \backslash B_{i}$. Then, the following jackknife histogram is a distribution estimator of $\tau_{n}\left(T_{n}-T\right)$ :

$$
H_{N}(x)=(n-\ell+1)^{-1} \sum_{i=1}^{n-\ell+1} 1\left\{\tau_{\ell} \ell^{-1}(n-\ell)\left(T_{n,-i}-T_{n}\right) \leq x\right\},
$$

where the $\tau_{\ell}$ is an appropriate normalizing constant.

- For the moving block bootstrap (MBB), we resample $k$ blocks from $\left\{B_{1}, B_{2}, \ldots, B_{n-\ell+1}\right\}$ with replacement and with equal probability for each block to be drawn. For simplicity, we assume $n=k \ell$, then a bootstrap resample is obtained by joining the blocks together in random order. The bootstrap statistic its defined by $T_{n}^{*}$ which is equal to the estimator $T_{n}$ evaluated in the bootstrap resample. Then, the bootstrap distribution:

$$
\operatorname{Pr}^{*}\left\{\tau_{n}\left(T_{n}^{*}-T_{n}\right) \leq x\right\}
$$

is a distribution estimator of $\tau_{n}\left(T_{n}-T\right)$.

Some works point out that in blockwise bootstrap a smooth transition between blocks could be preferable to random joining, see Carlstein et al. (1998). Also, Künsch (1989) shows that in MBJ it is better to downweight the block $B_{i}$ instead of a completed deletion. Those facts motivate the moving block resampling by missing values techniques proposed in Alonso et al. (2003). 
- In the moving missing block jackknife $\left(\mathrm{M}^{2} \mathrm{BJ}\right)$, we consider the deleted block $B_{i}$ as $\ell$ consecutive missing values and we estimated those observations taking into account the dependence structure of $\left\{X_{t}\right\}_{t \in \mathbb{Z}}$. Then the $i$-th $\mathrm{M}^{2} \mathrm{BJ}$ statistics $\widetilde{T}_{n,-i}$ is equal to the estimator $T_{n}$ evaluated in $\left(\mathbf{X} \backslash B_{i}\right) \cup \widehat{B}_{i}$, where $\widehat{B}_{i}$ denotes the estimate of $B_{i}$. Then, the following $\mathrm{M}^{2} \mathrm{BJ}$ histogram is a distribution estimator of $\tau_{n}\left(T_{n}-T\right)$ :

$$
\widetilde{H}_{N}(x)=(n-\ell+1)^{-1} \sum_{i=1}^{n-\ell+1} 1\left\{\tau_{\ell} \ell^{-1}(n-\ell)\left(\widetilde{T}_{n,-i}-T_{n}\right) \leq x\right\} .
$$

Notice that $\mathrm{M}^{2} \mathrm{BJ}$ statistics have the computational advantage of using the same functional form as $T_{n}$, while for MBJ statistics we should implement the calculations considering that $\ell$ observations are missing.

- In the moving missing block bootstrap $\left(\mathrm{M}^{2} \mathrm{BB}\right)$, as in $\mathrm{MBB}$, we have in each resample $k$ blocks $\left(B_{i_{1}}^{*}, B_{i_{2}}^{*}, \ldots, B_{i_{k}}^{*}\right)$. Let $\ell=b+m$, and we consider the $m$ last observations in each block as missing values. Thus, we will have $\mathrm{km}$ missing observations that will be replaced by their estimates. The $\mathrm{M}^{2} \mathrm{BB}$ statistic is defined by $\widetilde{T}_{n}^{*}$ which is equal to the estimator $T_{n}$ evaluated in the resulting resample. Then, the bootstrap distribution

$$
\operatorname{Pr}^{*}\left\{\tau_{n}\left(\widetilde{T}_{n}^{*}-T_{n}\right) \leq x\right\}
$$

is a distribution estimator of $\tau_{n}\left(T_{n}-T\right)$.

The $\mathrm{M}^{2} \mathrm{BB}$ scheme resemble to a block joining engine similar to the matched-block bootstrap of Carlstein et al. (1998).

In our case $T_{n}=\widehat{p}$, and the computational implementation of $\mathrm{M}^{2} \mathrm{BJ}$ and $\mathrm{M}^{2} \mathrm{BB}$ only requires additionally a missing values estimation method. We will use the generalized least square estimators proposed by Peña and Maravall (1991). The consistency of (2)-(5) estimators for this particular statistics is beyond of the scope of this paper. 
A problem related with blockwise resampling is the selection of block size $\ell$ (in ours case, $\ell$ and $k$ ). A general approach to solve this problem was proposed by Hall et al. (1995) for MBB distribution and variance estimators. This approach could be easily modified in order to select $\ell$ and $k$ for $\mathrm{M}^{2} \mathrm{BJ}$ and $\mathrm{M}^{2} \mathrm{BB}$, but it involves a discrete optimization in $\ell$ and $k$ that could be a computational disadvantage. Since in each bootstrap resample we have to estimate autoregressive models up to order $p_{\max }$, we use in the Monte Carlo study $\ell=2 p_{\max }, 3 p_{\max }$ and $4 p_{\max }$ (with $p_{\max }=n / 10$ as recommended Bhansali 1983) and $k=1$ in order to have a moderate number of missing values.

\subsection{The sieve exogenous order blockwise bootstrap}

Let $\left\{X_{t}\right\}_{t \in \mathbb{Z}}$ be a real valued, stationary process with expectation $\mathrm{E}\left[X_{t}\right]=$ $\mu_{X}$ that admits a one-sided infinite-order autoregressive representation:

$$
\sum_{j=0}^{+\infty} \phi_{j}\left(X_{t-j}-\mu_{X}\right)=\varepsilon_{t}, \quad \phi_{0}=1, t \in \mathbb{Z},
$$

with coefficients $\left\{\phi_{j}\right\}_{j=0}^{+\infty}$ satisfying $\sum_{j=0}^{+\infty} \phi_{j}^{2}<\infty$. This representation motivates the $\operatorname{AR}(\infty)$-sieve bootstrap, that was first proposed by Kreiss (1988) and extensions can be found in Bühlmann (1997) and Inoue and Kilian (2002).

The method proceeds as follows:

1. Given a sample $\left\{X_{1}, \ldots, X_{n}\right\}$, select the order $\widehat{p}$ of the autoregressive approximation by some information criterion (e.g. AIC, AICC or BIC).

2. Obtain the Yule-Walker estimates of the coefficients for the $p_{\max }+1$ autoregressive models: $\left(\widehat{\phi}_{1}^{(p)}, \widehat{\phi}_{2}^{(p)}, \ldots, \widehat{\phi}_{p}^{(p)}\right)$ for $1 \leq p \leq p_{\max }$, and $\widehat{\phi}_{0}^{(p)}=1$ for $0 \leq p \leq p_{\max }$.

3. Compute the residuals for the model with $p=\widehat{p}$ :

$$
\widehat{\varepsilon}_{t}=\sum_{j=0}^{\widehat{p}} \widehat{\phi}_{j}^{(\widehat{p})}\left(X_{t-j}-\bar{X}\right), \quad t \in(\widehat{p}+1, \ldots, n) .
$$


4. Define the empirical distribution functions of the centered residuals:

$$
\widehat{F}_{\widetilde{\varepsilon}}(x)=(n-\widehat{p})^{-1} \sum_{t=\widehat{p}+1}^{n} 1_{\left\{\widetilde{\varepsilon}_{t} \leq x\right\}},
$$

where $\tilde{\varepsilon}_{t}=\widehat{\varepsilon}_{t}-\widehat{\varepsilon}^{(\cdot)}$, and $\widehat{\varepsilon}^{(\cdot)}=(n-\widehat{p})^{-1} \sum_{t=\widehat{p}+1}^{n} \widehat{\varepsilon}_{t}$.

5. Draw a $\mathrm{M}^{2} \mathrm{BB}$ resample from the sample $\left\{X_{1}, \ldots, X_{n}\right\}$, and select the order $p^{*}$ of the autoregressive approximation (as in the step 1) for this resample.

6. Draw a resample $\varepsilon_{t}^{*}$ of i.i.d. observations from $\widehat{F}_{\widetilde{\varepsilon}}$.

7. Define $X_{t}^{*}$ by the recursion:

$$
\sum_{j=0}^{p^{*}} \widehat{\phi}_{j}^{\left(p^{*}\right)}\left(X_{t-j}^{*}-\bar{X}\right)=\varepsilon_{t}^{*}
$$

where the starting $p^{*}$ observations are equal to $\bar{X}$.

8. Compute the estimation of the autoregressive coefficients: $\left(\widehat{\phi}_{1}^{*}, \ldots, \widehat{\phi}_{p^{*}}^{*}\right)$, as in step 2.

9. Compute future bootstrap observations by the recursion:

$$
X_{T+h}^{*}=\bar{X}-\sum_{j=1}^{p^{*}} \widehat{\phi}_{j}^{*}\left(X_{T+h-j}^{*}-\bar{X}\right)+\varepsilon_{t}^{*},
$$

where $h>0$, and $X_{t}^{*}=X_{t}$, for $t \leq T$.

Finally, $F_{X_{T+h}^{*}}^{*}$ the bootstrap distribution of $X_{T+h}^{*}$ is used to approximate the unknown distribution of $X_{T+h}$ given the observed sample. As usual, a Monte Carlo estimate $\widehat{F}_{X_{T+h}^{*}}^{*}$ is obtained by repeating the steps 5 to $9 B$ times. The $(1-\alpha) \%$ prediction interval for $X_{T+h}$ is given by

$$
\left[Q^{*}(\alpha / 2), Q^{*}(1-\alpha / 2)\right]
$$

where $Q^{*}(\cdot)=\widehat{F}_{X_{T+h}^{*}}^{*-1}(\cdot)$ are the quantiles of the estimated bootstrap distribution. 


\section{Simulations results}

We compare the different sieve bootstrap approaches for the following models:

Model 1: $\left(1-0.75 B+0.5 B^{2}\right) X_{t}=\varepsilon_{t}$

Model 2: $X_{t}=\left(1-0.3 B+0.7 B^{2}\right) \varepsilon_{t}$.

Model 3: $\left(1+0.7 B-0.2 B^{2}\right) X_{t}=\varepsilon_{t}$

Model 4: $X_{t}=\left(1+0.7 B-0.2 B^{2}\right) \varepsilon_{t}$.

Model 1 was considered by Cao et al. (1997) and Model 2 by Pascual et al. (2001). Model 3 and 4 were considered by Alonso et al. (2004). As in those papers we used the following error distributions $F_{\varepsilon}$ : the standard normal, a shifted exponential distribution with zero mean and scale parameter equal to one, and a contaminated distribution $0.9 F_{1}+0.1 F_{2}$ with $F_{1} \sim \mathcal{N}(-1,1)$ and $F_{2} \sim \mathcal{N}(9,1)$. But, for sake of brevety, we only present the results for the standard normal. We take sample sizes $n=50$, and 100 , leads $h=1$ to $h=5$, and nominal coverage $1-\alpha=0.95$.

To compare the different prediction intervals, we use their mean coverage $\left(\bar{C}_{M}\right)$ and length $\left(\bar{L}_{M}\right)$, and the proportions of observations lying out to the left and to the right of the interval.

The different sieve bootstrap are denoted by:

S corresponds to the sieve bootstrap without introducing model uncertainty, i.e. the algorithm of Section 2 but omitting the step 5 and using $p^{*}=\widehat{p}$ in steps $7-9$.

EnS the endogenous sieve bootstrap using $\widehat{p}$ obtained by AICC (see section 2.1 of Alonso et al., 2004).

ExS1 the exogenous sieve bootstrap using the moving missing block bootstrap.

ExS2 the exogenous sieve bootstrap using the AICC information criterion (see section 2.2 of Alonso et al., 2004). 
Notice that the difference between $\mathrm{S}$ and the other sieve bootstrap procedures corresponds to the variability associated to model uncertainty. A Fortran implementation of procedure S can be found in Alonso (2004).

In Tables 1-4, we present the results for the four models, using the two sample sizes and Gaussian innovations, nominal coverage 95\%, and lead times $h=1$ and 5. For ExS1, we report the results with $\ell=3 p_{\max }$. The other possible combinations of parameters are available on request to the authors.

Table 1 Simulation results for Model 1, with Gaussian Errors.

\begin{tabular}{|c|c|c|c|c|c|}
\hline Lag & Sample & Method & $\bar{C}_{M}(\mathrm{se})$ & Cov. (b./a.) & $\bar{L}_{M}(\mathrm{se})$ \\
\hline $\mathrm{h}$ & $\mathrm{n}$ & Theoretical & $95 \%$ & $2.50 \% / 2.50 \%$ & 3.93 \\
\hline 1 & 50 & $\mathrm{~S}$ & $92.27(0.13)$ & $3.92 / 3.81$ & $3.83(0.02)$ \\
\hline & & EnS & $92.59(0.12)$ & $3.75 / 3.66$ & $3.87(0.02)$ \\
\hline & & ExS1 & $92.56(0.12)$ & $3.79 / 3.65$ & $3.85(0.02)$ \\
\hline & & ExS2 & $92.74(0.12)$ & $3.69 / 3.57$ & $3.88(0.02)$ \\
\hline & 100 & $\mathrm{~S}$ & $93.53(0.09)$ & $3.17 / 3.30$ & $3.88(0.01)$ \\
\hline & & EnS & $93.83(0.08)$ & $3.00 / 3.18$ & $3.92(0.01)$ \\
\hline & & ExS1 & $93.77(0.09)$ & $3.07 / 3.16$ & $3.92(0.01)$ \\
\hline & & ExS2 & $93.96(0.08)$ & $2.97 / 3.07$ & $3.96(0.01)$ \\
\hline $\mathrm{h}$ & $\mathrm{n}$ & Theoretical & $95 \%$ & $2.50 \% / 2.50 \%$ & 5.20 \\
\hline 5 & 50 & $\mathrm{~S}$ & $92.01(0.12)$ & $4.03 / 3.96$ & $4.86(0.02)$ \\
\hline & & EnS & $92.18(0.12)$ & $3.91 / 3.92$ & $4.88(0.02)$ \\
\hline & & ExS1 & $92.12(0.13)$ & $3.92 / 3.96$ & $4.87(0.02)$ \\
\hline & & ExS2 & $92.29(0.12)$ & $3.82 / 3.89$ & $4.91(0.02)$ \\
\hline & 100 & S & $93.47(0.09)$ & $3.27 / 3.26$ & $5.02(0.02)$ \\
\hline & & EnS & $93.66(0.08)$ & $3.19 / 3.15$ & $5.05(0.02)$ \\
\hline & & ExS1 & $93.71(0.08)$ & $3.17 / 3.12$ & $5.06(0.02)$ \\
\hline & & ExS2 & $93.93(0.08)$ & $3.08 / 2.99$ & $5.13(0.02)$ \\
\hline
\end{tabular}

NOTE: Standard error (se) are in parentheses.

For Models 1 and 3 with Gaussian errors, methods EnS, ExS1 and ExS2 have a better performance than $\mathrm{S}$ in terms of mean coverage and length for all sample sizes and lead times (up to a $0.4 \%$ more of mean coverage for Model 1 and $h=1$ and up to a 1.5\% more of mean coverage for Model 3 and $h=1$ ). If we use the following measure of the improvement by introducing model uncertainty of a procedure $M: \frac{\bar{C}_{M}-\bar{C}_{S}}{0.95-\bar{C}_{S}}$ where $\bar{C}_{S}$ is the mean coverage 
Table 2 Simulation results for Model 2, with Gaussian Errors.

\begin{tabular}{|c|c|c|c|c|c|}
\hline Lag & Sample & Method & $\bar{C}_{M}(\mathrm{se})$ & Cov. (b./a.) & $\bar{L}_{M}(\mathrm{se})$ \\
\hline $\mathrm{h}$ & $\mathrm{n}$ & Theoretical & $95 \%$ & $2.50 \% / 2.50 \%$ & 3.93 \\
\hline 1 & 50 & $\mathrm{~S}$ & $91.30(0.19)$ & $4.05 / 4.65$ & $3.96(0.02)$ \\
\hline & & EnS & $91.69(0.19)$ & $3.89 / 4.43$ & $4.03(0.02)$ \\
\hline & & ExS1 & $91.67(0.18)$ & $3.93 / 4.40$ & $4.00(0.02)$ \\
\hline & & ExS2 & $92.00(0.18)$ & $3.77 / 4.23$ & $4.00(0.02)$ \\
\hline & 100 & $\mathrm{~S}$ & $93.00(0.11)$ & $3.58 / 3.43$ & $3.93(0.01)$ \\
\hline & & EnS & $93.26(0.11)$ & $3.42 / 3.32$ & $3.97(0.01)$ \\
\hline & & ExS1 & $93.36(0.11)$ & $3.33 / 3.31$ & $3.96(0.01)$ \\
\hline & & ExS2 & $93.59(0.11)$ & $3.24 / 3.17$ & $3.99(0.01)$ \\
\hline $\mathrm{h}$ & $\mathrm{n}$ & Theoretical & $95 \%$ & $2.50 \% / 2.50 \%$ & 4.94 \\
\hline 5 & 50 & $\mathrm{~S}$ & $91.69(0.13)$ & $4.08 / 4.23$ & $4.63(0.02)$ \\
\hline & & EnS & $91.88(0.13)$ & $3.99 / 4.13$ & $4.64(0.02)$ \\
\hline & & ExS1 & $91.75(0.13)$ & $4.05 / 4.20$ & $4.62(0.02)$ \\
\hline & & ExS2 & $91.93(0.13)$ & $3.99 / 4.08$ & $4.63(0.02)$ \\
\hline & 100 & S & $93.03(0.09)$ & $3.51 / 3.46$ & $4.75(0.01)$ \\
\hline & & EnS & $93.20(0.09)$ & $3.45 / 3.35$ & $4.77(0.01)$ \\
\hline & & ExS1 & $93.21(0.09)$ & $3.38 / 3.42$ & $4.76(0.01)$ \\
\hline & & ExS2 & $93.30(0.09)$ & $3.33 / 3.37$ & $4.76(0.01)$ \\
\hline
\end{tabular}

NOTE: Standard error (se) are in parentheses.

of the bootstrap method without including model uncertainty. Then the increases are up to $17.2 \%$ for Model 1 and up to $42.3 \%$ for Model 3 .

We obtain similar results for Models 2 and 4 . Notice that in these case, the sieve approach never uses the correct model. We observe that, for $h=$ $1, \mathrm{~S}$ method is outperformed by all sieve approaches that include model variability (up to a $0.7 \%$ more of mean coverage for Model 2 and up to a $2.2 \%$ more of mean coverage for Model 3). The improvements are up to 18.9\% for Model 1 and up to $52.6 \%$ for Model 3 .

In all models ExS1 have similar results to the previous proposed ExS2. It should be remarked that none of the procedures that include model variability is superior to the others. The EnS and ExS2 procedures have the advantage of a simpler implementation. ExS2 is computationally better than EnS since it does not select a model in each resample. In the other hand the blockwise bootstrap is applicable to more general dependence structures. 
Table 3 Simulation results for Model 3, with Gaussian Errors.

\begin{tabular}{|c|c|c|c|c|c|}
\hline Lag & Sample & Method & $\bar{C}_{M}(\mathrm{se})$ & Cov. (b./a.) & $\bar{L}_{M}(\mathrm{se})$ \\
\hline $\mathrm{h}$ & $\mathrm{n}$ & Theoretical & $95 \%$ & $2.50 \% / 2.50 \%$ & 3.94 \\
\hline 1 & 50 & $\mathrm{~S}$ & $91.29(0.16)$ & $4.32 / 4.40$ & $3.76(0.02)$ \\
\hline & & EnS & $92.69(0.13)$ & $3.63 / 3.68$ & $3.93(0.02)$ \\
\hline & & ExS1 & $92.81(0.12)$ & $3.55 / 3.63$ & $3.90(0.02)$ \\
\hline & & ExS2 & $92.86(0.13)$ & $3.55 / 3.58$ & $3.95(0.02)$ \\
\hline & 100 & $\mathrm{~S}$ & $93.08(0.11)$ & $3.48 / 3.44$ & $3.85(0.01)$ \\
\hline & & EnS & $93.69(0.09)$ & $3.16 / 3.15$ & $3.92(0.01)$ \\
\hline & & ExS1 & $93.93(0.09)$ & $3.14 / 2.94$ & $3.93(0.01)$ \\
\hline & & ExS2 & $93.80(0.09)$ & $3.08 / 3.12$ & $3.94(0.01)$ \\
\hline $\mathrm{h}$ & $\mathrm{n}$ & Theoretical & $95 \%$ & $2.50 \% / 2.50 \%$ & 6.45 \\
\hline 5 & 50 & $\mathrm{~S}$ & $92.14(0.19)$ & $3.91 / 3.95$ & $6.36(0.04)$ \\
\hline & & EnS & $92.93(0.18)$ & $3.54 / 3.53$ & $6.50(0.04)$ \\
\hline & & ExS1 & $93.41(0.17)$ & $3.23 / 3.36$ & $6.57(0.04)$ \\
\hline & & ExS2 & $92.37(0.18)$ & $3.85 / 3.78$ & $6.35(0.03)$ \\
\hline & 100 & $\mathrm{~S}$ & $93.22(0.13)$ & $3.42 / 3.36$ & $6.35(0.03)$ \\
\hline & & EnS & $93.85(0.12)$ & $3.09 / 3.07$ & $6.49(0.03)$ \\
\hline & & ExS1 & $94.20(0.11)$ & $2.92 / 2.88$ & $6.53(0.03)$ \\
\hline & & ExS2 & $93.58(0.12)$ & $3.22 / 3.20$ & $6.39(0.03)$ \\
\hline
\end{tabular}

NOTE: Standard error (se) are in parentheses.

Table 4 Simulation results for Model 4, with Gaussian Errors.

\begin{tabular}{|c|c|c|c|c|c|}
\hline Lag & Sample & Method & $\bar{C}_{M}(\mathrm{se})$ & Cov. (b./a.) & $\bar{L}_{M}(\mathrm{se})$ \\
\hline $\mathrm{h}$ & $\mathrm{n}$ & Theoretical & $95 \%$ & $2.50 \% / 2.50 \%$ & 3.93 \\
\hline 1 & 50 & $\mathrm{~S}$ & $90.82(0.18)$ & $4.62 / 4.56$ & $3.74(0.02)$ \\
\hline & & EnS & $92.60(0.12)$ & $3.73 / 3.67$ & $3.88(0.02)$ \\
\hline & & ExS1 & $92.80(0.13)$ & $3.68 / 3.53$ & $3.90(0.02)$ \\
\hline & & ExS2 & $93.02(0.12)$ & $3.52 / 3.46$ & $3.92(0.02)$ \\
\hline & 100 & $\mathrm{~S}$ & $93.05(0.11)$ & $3.54 / 3.41$ & $3.83(0.01)$ \\
\hline & & EnS & $93.63(0.09)$ & $3.27 / 3.10$ & $3.89(0.01)$ \\
\hline & & ExS1 & $93.85(0.08)$ & $3.00 / 3.15$ & $3.93(0.01)$ \\
\hline & & ExS2 & $93.83(0.09)$ & $3.15 / 3.02$ & $3.91(0.01)$ \\
\hline $\mathrm{h}$ & $\mathrm{n}$ & Theoretical & $95 \%$ & $2.50 \% / 2.50 \%$ & 4.87 \\
\hline 5 & 50 & $\mathrm{~S}$ & $93.48(0.12)$ & $3.30 / 3.23$ & $4.78(0.02)$ \\
\hline & & EnS & $93.84(0.11)$ & $3.13 / 3.02$ & $4.85(0.02)$ \\
\hline & & ExS1 & $94.31(0.11)$ & $2.79 / 2.90$ & $4.94(0.02)$ \\
\hline & & ExS2 & $94.24(0.11)$ & $2.90 / 2.86$ & $4.91(0.02)$ \\
\hline & 100 & $\mathrm{~S}$ & $94.05(0.09)$ & $2.98 / 2.97$ & $4.80(0.01)$ \\
\hline & & EnS & $94.37(0.08)$ & $2.81 / 2.82$ & $4.85(0.01)$ \\
\hline & & ExS1 & $94.79(0.07)$ & $2.60 / 2.61$ & $4.92(0.02)$ \\
\hline & & ExS2 & $94.56(0.08)$ & $2.73 / 2.71$ & $4.89(0.02)$ \\
\hline
\end{tabular}

NOTE: Standard error (se) are in parentheses. 


\section{Conclusion}

It has been shown by Masarotto (1990) and Grigoletto (1998) that if the order of the AR is unknown, but finite, it can obtained prediction intervals by bootstrap incorporating the sampling variability of $\widehat{p}$ with better coverage probabilities than those produced by standard bootstrap procedures. Their approaches could be affected by the selected order $\widehat{p}$. In Alonso et al. (2004) a sieve endogenous (and exogenous) order bootstrap have been proposed and a simulation experiment have shown that both procedures improve the standard sieve bootstrap. In this paper we have proposed an alternative method based on moving blocks bootstrap for introducing the model selection variability in the prediction intervals. Monte Carlo simulations show that the proposed procedure provide comparable coverage results than previous methods in general cases meanwhile its less dependent on the initial selected order.

Acknowledgements This research was partially supported by the Plan Nacional I+D+I project BEC 2002-2054 and by the Fundación BBVA.

\section{References}

1. Akaike, H. (1969) Fitting autoregressive models for prediction, Ann. Inst. Statist. Math., 21, 243-247.

2. Akaike, H. (1973) Information theory and an extension of the maximum likelihood principle, 2nd International Symposium on Information Theory, B.N. Petrov, and F. Csaki, Eds., Akademiai Kiado, Budapest, 267-281.

3. Alonso, A.M. (2004) A Fortran routine for sieve bootstrap prediction intervals, J. Modern Appl. Statist. Methods, 3, 239-249.

4. Alonso, A.M., Peña, D. and Romo, J. (2002) Forecasting time series with sieve bootstrap, J. Statist. Plann. Inference, 100, 1-11.

5. Alonso, A.M., Peña, D. and Romo, J. (2003) Resampling time series using missing values techniques, Ann. Inst. Statist. Math., 55, 765-796.

6. Alonso, A.M., Peña, D. and Romo, J. (2004) Introducing model uncertainty in time series bootstrap, Statist. Sinica, 14, 155-174.

7. Bhansali, R.J. (1983) A simulation study of autoregressive and window estimators of the inverse autocorrelation function, Appl. Statist., 32 141-149.

8. Bhansali, R.J. (1993) Order selection for linear time series models: A review. In: Developments in Time Series Analysis, T. Subba Rao, ed., Chapman and Hall, London, 50-66. 
9. Box, G.E.P, Jenkins, G.M. and Reinsel, G.C. (1994) Time Series Analysis: Forecasting and Control, Prentice Hall. Englewood.

10. Bühlmann, P. (1997) Sieve bootstrap for time series, Bernoulli, 8, 123-148.

11. Cao, R., Febrero-Bande, M., González-Manteiga, W., Prada-Sánchez, J.M. and García-Jurado, I. (1997) Saving computer time in constructing consistent bootstrap prediction intervals for autoregressive processes, Comm. Statist. Simulation Comput., 26, 961-978.

12. Carlstein, E., Do, K., Hall, P., Hesterberg, T. and Künsch, H. R. (1998) Matched-block bootstrap for dependent data, Bernoulli, 4, 305-328.

13. Chatfield, C. (1993) Calculating interval forecasts, J. Bus. Econom. Statist., 11, 121-135.

14. Chatfield, C. (1996) Model uncertainty and forecast accuracy, J. Forecasting, 15, 495-508.

15. Grigoletto, M. (1998) Bootstrap prediction intervals for autoregresions: some alternatives, Intern. J. Forecasting, 14, 447-456.

16. Hall, P., Horowitz, J.L. and Jing, B.Y. (1995) On blocking rules for the bootstrap with dependent data, Biometrika, 82, 561-574.

17. Hurvich, C.M. and Tsai, C.L. (1989) Regression and time series model selection in small samples, Biometrika, 76, 297-307.

18. Inoue, A. and Kilian, L. (2002). Bootstrapping smooth functions of slope parameters and innovations variance in $\operatorname{VAR}(\infty)$ models. Internat. Econom. Rev. 43, 309-331.

19. Kilian, L. (1998) Accounting for lag uncertainty in autoregressions: The endogenous lag order bootstrap algorithhm, J. Time Ser. Anal., 19, 531-548.

20. Kreiss, J.-P. (1988) Asymptotical Inference of Stochastic Processes, Habilitationsschrift, Universität Hamburg.

21. Künsch, H.R. (1989) The jackknife and the bootstrap for general stationary observations, Ann. Statist., 17, 1217-1241.

22. Liu, R.Y. and Singh, K. (1992) Moving blocks jackknife and bootstrap capture week dependence, In: Exploring the Limits of Bootstrap, R. Lepage and L. Billard eds., 225-248, Wiley, New York.

23. Masarotto, G. (1990) Bootstrap prediction intervals for autoregressions, Intern. J. Forecasting, 6, 229-239.

24. Pascual, L., Romo, J. and Ruiz, E. (2001) Effects of parameter estimation on prediction densities: a bootstrap approach. Intern. J. Forecasting 17, 83-103.

25. Peña, D. and Maravall, A. (1991) Interpolation, outliers, and inverse autocorrelations, Comm. Statist. Theory Methods, 20, 3175-3186.

26. Stine, R.A. (1987) Estimating properties of autoregressive forecasts, J. Amer. Statist. Assoc., 82, 1072-1078.

27. Thombs, L.A. and Schucany, W.R. (1990) Bootstrap prediction intervals for autoregression, J. Amer. Statist. Assoc., 85, 486-492.

28. Schwarz, G. (1978) Estimating the dimension of a model, Ann. Statist., 6, 461-464. 